\title{
Caracterização epidemiológica das reações transfusionais imediatas em um hospital
}

\section{público de ensino}

\author{
Epidemiological characterization of immediate transfusion reactions in a public teaching hospital \\ Caracterización epidemiológica de las reacciones transfusionales inmediatas en un hospital público
}

docente

Recebido: 30/05/2021 | Revisado: 06/07/2021 | Aceito: 11/07/2021 | Publicado: 21/07/2021

\author{
Aline Bezerra Silva \\ ORCID: https://orcid.org/0000-0003-4628-6844 \\ Universidade Federal de Sergipe, Brasil \\ E-mail: aline.bs@ebserh.gov.br \\ Adicinéia Aparecida de Oliveira \\ ORCID: https://orcid.org/0000-0002-1551-1992 \\ Universidade Federal de Sergipe, Brasil \\ E-mail: adicineia@ufs.br \\ Juliana de Oliveira Musse Silva \\ ORCID: https://orcid.org/0000-0003-1713-6791 \\ Universidade Tiradentes, Brasil \\ E-mail: julimusse@ hotmail.com \\ Gleyce Kelly de Brito Brasileiro Santos \\ ORCID: https://orcid.org/0000-0003-4578-5799 \\ Universidade Federal de Sergipe, Brasil \\ E-mail: gkbsantos@hotmail.com \\ Rafael Braga Esteves \\ ORCID: https://orcid.org/0000-0003-4604-6840 \\ Universidade de São Paulo, Brasil \\ E-mail: rafael.braga.esteves@alumni.usp.br \\ Ângela Maria da Silva \\ ORCID: https://orcid.org/0000-0001-9330-537X \\ Universidade Federal de Sergipe, Brasil \\ E-mail: angela.silva910@gmail.com
}

\begin{abstract}
Resumo
Objetivos: Caracterizar as Reações Transfusionais (RT) imediatas e analisar os meios para diagnóstico dessas reações. Metodologia: Trata-se de uma pesquisa com delineamento longitudinal retrospectivo, exploratório e descritivo, realizado em um Hospital Universitário Público de Aracaju - SE, Brasil. Todos os aspectos éticos foram respeitados aprovado pelo Comitê de Ética em Pesquisa da Universidade Federal de Sergipe, a coleta de dados ocorreu durante o período de 2019 e 2020. Os dados relativos as reações transfusionais foram extraídas do sistema de notificação do hospital e foram caracterizados segundo protocolos nacionais e internacionais, quanto à imputabilidade de uma Reação Transfusional com critérios clínicos, laboratoriais e com o vínculo temporal. A análise estatística realizada dos dados foi descritiva e inferencial de acordo com as variáveis e objetivos dos pesquisadores. Resultados: A data do incidente transfusional e a notificação no sistema conferiu um tempo médio de 3,91 dias para que os profissionais notificassem as RT. O histórico transfusional não foi preenchido em 85\% (67) das notificações. A possibilidade de recidivas de RT nesses pacientes ocorreu entre 1 a 18 dias do evento inicial e apenas $27 \%(\mathrm{n}=3$ ) estavam em unidades distintas. Considerações finais: As reações transfusionais quando notificadas demonstraram uma fonte rica para um cuidado mais adequado para pacientes submetidos ao tratamento transfusional com hemocomponentes.
\end{abstract}

Palavras-chave: Reação transfusional; Transfusão sanguínea; Protocolos; Segurança transfusional; Estudos epidemiológicos.

\begin{abstract}
Objectives: To characterize immediate Transfusion Reactions (RT) and analyze the means for diagnosing these reactions. Methodology: This is a research with a retrospective, exploratory and descriptive longitudinal design, carried out at a Public University Hospital in Aracaju - SE, Brazil. All ethical aspects were respected approved by the Research Ethics Committee of the Federal University of Sergipe, data collection took place between 2019 and 2020. Data on transfusion reactions were extracted from the hospital's notification system and were characterized according to national and international protocols, as to the imputability of a Transfusional Reaction with clinical and laboratory criteria and with the temporal bond. The statistical analysis performed on the data was descriptive and inferential according to the researchers' variables and objectives. Results: The date of the transfusion incident and the notification
\end{abstract}


in the system provided an average time of 3.91 days for professionals to notify the TRs. The transfusion history was not completed in $85 \%$ (67) of the notifications. The possibility of RT recurrences in these patients occurred between 1 to 18 days after the initial event and only $27 \%(n=3)$ were in different units. Final considerations: Transfusion reactions when notified demonstrate a rich source for more adequate care for patients undergoing transfusion treatment with blood components.

Keywords: Transfusion reaction; Blood transfusion; Protocols; Blood safety; Epidemiologic studies.

\section{Resumen}

Objetivos: Caracterizar las Reacciones Transfusionales (RT) inmediatas y analizar los medios para diagnosticar estas reacciones. Metodología: Se trata de una investigación con un diseño longitudinal retrospectivo, exploratorio y descriptivo, realizada en un Hospital Universitario Público de Aracaju - SE, Brasil. Todos los aspectos éticos fueron respetados aprobados por el Comité de Ética en Investigación de la Universidad Federal de Sergipe, la recolección de datos se realizó entre 2019 y 2020. Los datos sobre reacciones transfusionales se extrajeron del sistema de notificación del hospital y se caracterizaron según protocolos nacionales e internacionales, como la imputabilidad de una Reacción Transfusional con criterios clínicos y de laboratorio y con el vínculo temporal. El análisis estadístico realizado sobre los datos fue descriptivo e inferencial según las variables y objetivos de los investigadores. Resultados: La fecha del incidente de transfusión y la notificación en el sistema proporcionaron un tiempo promedio de 3,91 días para que los profesionales notificaran a los TR. El historial de transfusiones no se completó en el $85 \%$ (67) de las notificaciones. La posibilidad de recidivas de la RT en estos pacientes ocurrió entre 1 y 18 días después del evento inicial y solo el $27 \%(\mathrm{n}=3)$ estaban en diferentes unidades. Consideraciones finales: Las reacciones a las transfusiones, cuando se notifican, demuestran una fuente rica para una atención más adecuada para los pacientes sometidos a tratamiento de transfusión con componentes sanguíneos.

Palabras clave: Reacción a la transfusión; Transfusión sanguínea; Protocolos; Seguridad de la sangre; Estudios epidemiológicos.

\section{Introdução}

Apesar da eficiência do processo de transfusão e melhorias graduais na condição clínica dos pacientes transfundidos, o procedimento pode acarretar riscos de cunho imunológico e não imunológico (Lee et al., 2019). As reações transfusionais (RT) são provenientes das relações direta de hemocomponente (HC), considerando o processo de armazenamento e liberação de mediadores pró-inflamatórios, oriundo de um doador, pode causar problemas por incidentes durante o ato transfusional, reações decorrentes da constituição biofisiológica e genéticas de um paciente (Kim \& Na, 2015). Outros problemas relacionados às RT são o aumento dos custos hospitalares e taxas de morbimortalidade, os problemas citados poderiam ser minimizados, com pequenas intervenções (Rodrigues \& Ribeiro, 2021).

No Brasil, o sistema de notificação da RT é compulsória desde 2010, de acordo com a RDC 57/2010, porém mesmo depois de muitos anos da criação desta RDC, verifica-se que as notificações ainda são pouco realizadas (Agência Nacional de Vigilância Sanitária - ANVISA, 2016; Sobral, Göttems, \& Santana, 2020). A literatura científica especializada demonstra um número relevantes de estudos, que trouxeram pouco impacto, inovação na área e o problema central deste artigo (Nazário, Barancelli, Gandolfi, Marcondes, \& Lilian Moura de Lima Spagnolo, 2019; Ribeiro et al., 2020; Santos, Teixeira, Pereira, \& Silva, 2020; Veiga \& Rojas, 2018).

A subnotificação das RT pode estar relacionada a diversos fatores como listados na sequência: ausência de informações sobre o ato transfusional e o percurso; falhas do monitoramento sistemático da transfusão; insegurança da equipe de saúde em correlacionar as manifestações clínicas ao uso de hemocomponentes ou ao desconhecimento da propedêutica específica para cada RT (Sobral, Göttems, \& Santana, 2020). Ainda neste sentido, um estudo realizado nos Estados Unidos da América, em 2015 mostrou que a ausência do repasse das informações dos setores sobre a possibilidade de RT para à Agência Transfusional pode alcançar até 75\% dos eventos (Gehrie, Hendrickson, \& Tormey, 2015).

O presente estudo sobre reações transfusionais é justificado devido o ineditismo e lacuna de conhecimento na literatura científica relacionadas à identificação de diagnósticos, usando a comparação de dados secundários ajustados aos dados, principalmente ao aspecto da análise de combinação de meios tornando possível diagnosticar uma RT. Os artigos sobre reações transfusionais descrevem diagnósticos se este foi registrado apenas. Ainda identificar diagnósticos pode evitar as 
recidivas dos pacientes que necessitam de transfusão de hemocomponentes, como pode se verificar em diversos estudos (Bosboom et al., 2018; Coberly \& Booth, 2016).

Diante do exposto, o presente artigo teve como objetivos caracterizar as reações transfusionais imediatas e analisar os meios para identificação de diagnósticos deste evento.

\section{Metodologia}

Trata-se de uma pesquisa com delineamento longitudinal retrospectivo, exploratório e descritivo, realizado em um Hospital Universitário Público de Aracaju - SE, Brasil. O presente artigo faz parte de um projeto maior intitulado: "Desenvolvimento de um protocolo integrado com sistema de alerta para o paciente em uso de hemocomponentes", aprovado pelo Comitê de Ética em Pesquisa da Faculdade de Enfermagem da Universidade Federal de Sergipe em junho de 2019, de acordo com o protocolo CAAE 33338720400005546 , a coleta de dados ocorreu durante o período de 2019 e 2020 . O projeto seguiu todos os preceitos éticos para investigações envolvendo seres humanos, especialmente os que estão descritos na Resolução n. 466/2012 (Brasil, 2012).

A amostra do estudo foi composta inicialmente restando 82 notificações identificadas como casos suspeitos de RT. Deste total foram excluídas 3 (três) pelos motivos: (1) por não apresentar o vínculo temporal, (2) a sintomatologia do paciente era prévia à transfusão e (3) não caracterizou febre. Para caracterizar uma reação transfusional febril é necessário o sinal de febre com parâmetros específicos como, é a elevação da temperatura em $1^{\circ} \mathrm{C}$ acima do valor de base ou acima de $38^{\circ} \mathrm{C}$, conforme é descrito em manuais especializados na área (Canadian Blood Services, 2017; Center for Disease Control/National Healthcare Safety Network, 2021; International Society of Blood Transfusion \& International Haemovigilance Network, 2019; Serious Hazards of Transfusion, 2019).

A instituição hospitalar, onde foi coletado os dados, incorporou a hemovigilância à gerência de risco no ano de 2014, através do sistema intitulado:" Vigilância em Saúde e Gestão de Riscos Assistenciais Hospitalares - VIGIHOSP”. Esse sistema era utilizado para notificar as RT, registro realizados por qualquer profissional, paciente, acompanhante ou estudante que apenas os integrantes da Unidade de Gestão Relacionados aos Aspectos Assistenciais (UGRA) tem acesso, posteriormente as informações eram enviadas para a Agência Transfusional (AT). Ainda no sentido de explicar o fluxo das informações sobre RT no VIGHOSP, para que fosse investigada o evento e encaminhada para a ANVISA, era necessário também o preenchimento da Ficha de Notificação e Investigação de Incidentes Transfusionais (FIT) que apenas os médicos podem fazê-lo. Esses dados eram compilados e encaminhados para o Serviço de Controle de Infecções Relacionadas à Assistência à Saúde (SCIRAS) para que a investigação ocorresse junto aos responsáveis da AT.

As informações não constaram desde 2014 até o ano de 2016 quando iniciaram as primeiras notificações e, por isso esse ano foi considerado como piloto sendo excluído da amostra. No período de 2017 a 2020 foram realizadas 4187 transfusões de hemocomponentes, segundo dados da VIGIHOSP. No estudo, os dados foram agrupados de acordo com as frequências, que foram comparadas para a confirmação do número de RT, na sequência foi apresentado o processo descrito, anteriormente (Figura 1): 
Figura 1 - Confirmação de RT de uma amostra de transfusões realizadas entre 2017 e 2020.

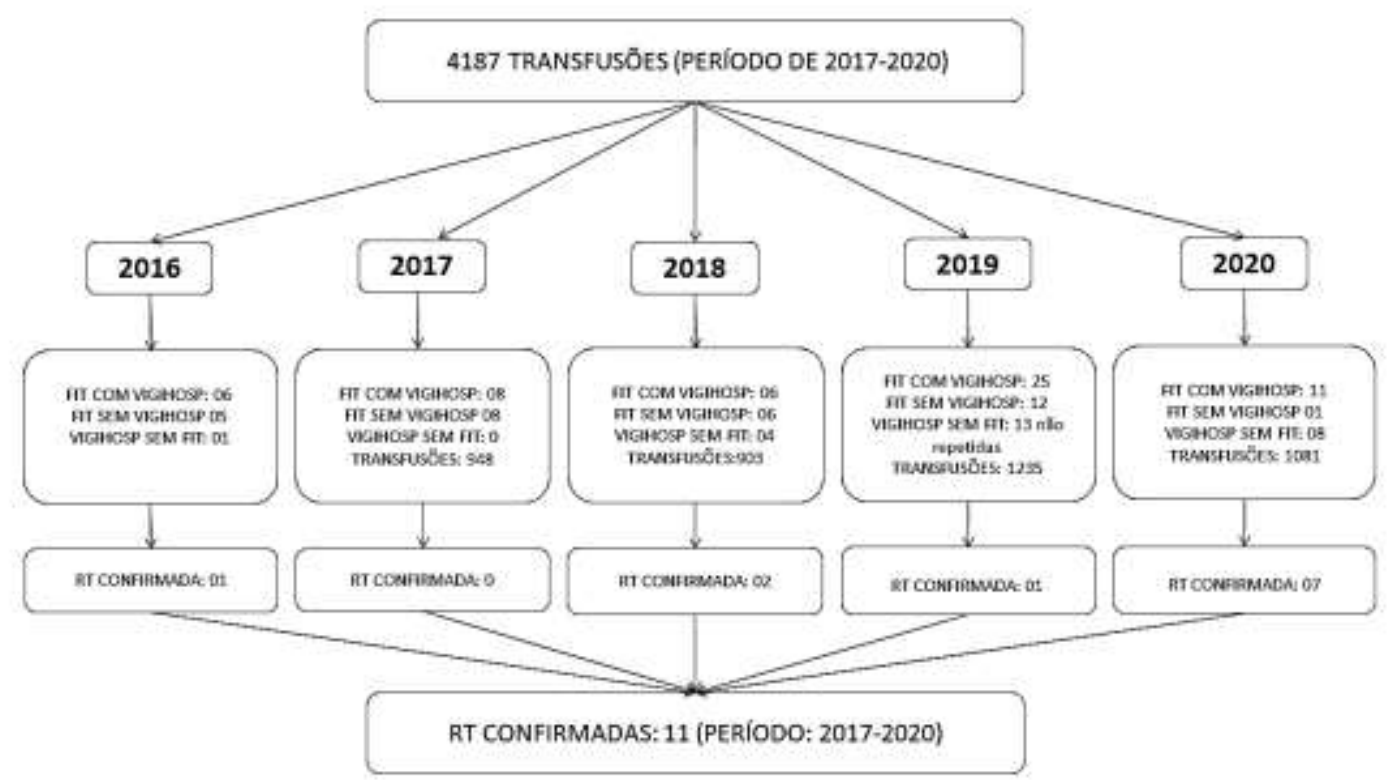

Nota: para a confirmação da reação transfusional, como apresentado na figura acima foi utilizado os dados do VIGIHOSP: Vigilância em Saúde e Gestão de Riscos Assistenciais Hospitalares, confrontados com as informações das FIT - Ficha de Notificação e Investigação de Incidentes Transfusionais, obtidas pelo Anvisa. Fonte: Autores (2021).

Os relatórios foram categorizados conforme as diretrizes de imputabilidade e a relação entre a transfusão e o evento adverso considerando manuais nacionais e internacionais (Agência Nacional de Vigilância Sanitária - ANVISA, 2015; Agence nationale de sécurité du medicament - ANSM, 2018; Canadian Blood Services - CBS, 2017; Center for Disease Control/National Healthcare Safety Network - CDC/NHSN, 2018, 2021; Instituto Português do Sangue e da Transplantação IPST, 2016, 2019; International Society of Blood Transfusion/International Haemovigilance Network - ISBT/IHN, 2019; Serious Hazards of Transfusion - SHOT, 2019): dessa forma, entende-se como RT confirmada, quando a investigação concluiu que há evidências claras (quadro clínico, laboratorial e vínculo temporal), sem qualquer dúvida acerca da correlação com a transfusão. As reações que não apresentem relação com a transfusão são consideradas como descartadas. E, quando a investigação apresenta evidências (quadro clínico, laboratorial e vínculo temporal), que indicam a correlação com a transfusão, mas há dúvidas para sua confirmação é dita como provável. A RT possível trata-se de uma investigação que indica a correlação dos sinais e sintomas que podem estar associadas a outras causas, mas a correlação com a transfusão não pode ser descartada. Quando à causalidade com a transfusão não pode ser excluída é referendada como improvável. E, sem evidências suficientes nem para confirmar nem para descartar é declarada como inconclusiva.

Outra classificação feita com base na gravidade da RT associado ao nível de dano ou incapacidade depois de uma RT ao paciente é proposta pela ANVISA e é classificada do Grau 1 a Grau 4 (Agência Nacional de Vigilância Sanitária ANVISA, 2015): Grau 1 (não grave): ausência de risco à vida, sem danos permanentes de órgãos ou função; Grau 2 (moderado): quando o paciente obteve dano com prejuízo das suas atividades decorrentes da transfusão que causou hospitalização ou deficiência ou incapacidade persistente com intervenção médica ou cirúrgica para evitar danos permanentes em órgão ou função; Grau 3 (risco de vida): ameaça grave à vida que necessitou de intervenção importante após a transfusão (vasopressores, intubação, transferência para a terapia intensiva) para evitar incapacidade de uma função do corpo ou evitar a morte; Grau 4 (óbito): o receptor morreu após uma reação adversa à transfusão.

No contexto do levantamento da imputabilidade quanto ao lapso temporal, foi veiculado às RT conforme a classificação elencada por diretrizes: Center for Disease Control/National Healthcare Safety Network, (2018, 2021); Vlaar et al., (2019); International Society of Blood Transfusion \& International Haemovigilance Network, (2019); Instituto Português 
do Sangue e da Transplantação, (2016). Assim sendo, as Reações Transfusionais Imediatas consideradas com nexo causal no contexto temporal são definidas no Quadro 1, a seguir:

Quadro 1 - Lapso temporal em relação a RT Aracaju, SE - Brasil, 2017 a 2020.

\begin{tabular}{|l|l|}
\hline Reações Transfusionais Imediatas & $\begin{array}{l}\text { Lapso temporal e associação com sinais e sintomas e } \\
\text { sinais vitais (Imputabilidade) }\end{array}$ \\
\hline Reação Alérgica (RALG) & $\begin{array}{l}4 \text { horas durante a transfusão ou após a cessação da } \\
\text { transfusão e sem outra evidência de correlação com os } \\
\text { riscos de reação alérgica a drogas, alimentação e ambiente. }\end{array}$ \\
\hline Reação Hipotensiva (HIPOT) & $\begin{array}{l}15 \text { min após o início da transfusão e responde rapidamente } \\
\text { após a suspensão da transfusão e tratamento (no intervalo } \\
\text { de } 10 \text { min.). }\end{array}$ \\
\hline Reação Febril não Hemolítica (RFNH) & $\begin{array}{l}4 \text { horas durante a transfusão ou após a cessação da } \\
\text { transfusão e sem outras condições que justifiquem os sinais } \\
\text { e sintomas. A febre é definida para a transfusão com a } \\
\text { elevação da temperatura em 1 }{ }^{\circ} \text { C acima do valor de base ou } \\
\text { pré-transfusional ou acima de 38 }\end{array}$ \\
\hline Reação Febril Hemolítica (RFH) & $\begin{array}{l}\text { Durante ou no intervalo das 24 horas da cessação da } \\
\text { transfusão. }\end{array}$ \\
\hline Sobrecarga Volêmica Associada à Transfusão (TACO) & $\begin{array}{l}\text { Realização de Raio x que comprove Insuficiência Cardíaca } \\
\text { Esquerda de origem cardiogênica na janela de } 12 \text { horas. }\end{array}$ \\
\hline Lesão Pulmonar Relacionada à Transfusão (TRALI) & $\begin{array}{l}\text { Realização de Raio x que comprove Insuficiência Cardíaca } \\
\text { Esquerda de origem não cardiogênica na janela de 06 horas. }\end{array}$ \\
\hline Dispnéia associada à Transfusão (TAD) & $\begin{array}{l}\text { Ocorre no período de 24 horas após a interrupção da } \\
\text { transfusão. }\end{array}$ \\
\hline
\end{tabular}

Fonte: Autores (2021).

Os dados coletados foram codificados e tabulados em uma planilha do software da Microsoft Excel $365^{\circledR}$, na sequência esses dados foram exportados para o Statistical Package for the Social Sciences (SPSS) versão 25. Procedeu-se a análise dos dados por meio da estatística descritivas dos itens que compõem o VIGIHOSP, sendo realizadas análises mais detalhadas como medidas de distribuição de frequências, número absolutos e percentuais, mínimo e máximos, medidas de tendência central e de dispersão para a estatística descritiva. Para a análise de resultados, utilizou-se a distribuição dos valores individuais originários de pontos de uma mesma fonte. O intervalo de confiança utilizado foi padronizado em $95 \%$ para apresentar significância estatística p<0,05 (Marconi \& Lakatos, 2003).

Para realizar a comparação das reações transfusionais e do tipo de hemocomponente utilizado com a idade, o tempo de infusão e o tempo de notificação, foi necessário a utilização do teste de normalidade Kolgomorov-Smirnov com Correlação de Lilliefors, para os grupos com um N maior ou igual que 20 e Shapiro-Wilk grupos menores com 20, com o intuito de avaliar a distribuição amostral. Quando agrupadas, apresentaram distribuição não paramétrica, selecionou-se o teste de Kruskal-Wallis a fim de avaliar a distribuição dos escores numéricos em detrimento aos grupos. Ao contrário das variáveis que quando agrupada apresentaram distribuição paramétrica, por isso optou-se o teste de Análise de Variância (ANOVA) a fim de avaliar a distribuição dos escores numéricos em detrimento aos grupos.

Ademais, também foi utilizado o teste de normalidade Kolgomorov-Smirnov com Correlação de Lilliefors a fim de definir os limites do intervalo entre a notificação da RT e o início da investigação, bem como o tempo de conclusão. Ao observar a distribuição heterogênea das variáveis, utilizou-se o teste Tau-b de Kendall a fim de correlacionar ambas variáveis numéricas e definir a força de correlação e a existência de relação entre ambas, analisando a força da correlação por meio do (Quadro 2): 
Quadro 2 - Força da correlação entre variáveis numéricas.

\begin{tabular}{|l|l|}
\hline Medida da Correlação & Interpretação \\
\hline 0,90 a $1,00(-0,90$ a $-1,00)$ & Correção muito forte positiva ou negativa \\
\hline 0,70 a $0,90(-0,70$ a $-0,90)$ & Correlação forte positiva ou negativa \\
\hline 0,50 a $0,70(-0,50$ a $-0,70)$ & Correlação moderada positiva ou negativa \\
\hline 0,30 a $0,50(-0,30$ a $-0,50)$ & Correlação fraca positiva ou negativa \\
\hline 0,00 a $0,30(0,00$ a $-0,30)$ & Correlação desprezível positiva ou negativa \\
\hline
\end{tabular}

Fonte: Adaptado de Hinkle, Wiersma, \& Jurs, (2003).

Utilizou-se os testes Exato de Fisher (para comparação quadráticas) e Qui-quadrado de Pearson (para comparações não quadráticas) a fim de avaliar a distribuição das variáveis. Foi utilizado também o índice de Kappa com o intuito de avaliar a existência de repetições entre as variáveis "coleta de amostras" e "coleta de uma amostra".

\section{Resultados e Discussão}

No período do estudo, houve 4187 transfusões com 79 notificações por meio do sistema VIGIHOSP e 88 vias de FIT com um total de 11 RT confirmadas. Quanto aos pacientes transfundidos, não houve equiparação entre os sexos, sendo a maioria do sexo feminino com 55,7\% ( $\mathrm{n}=44$ ), cor parda 64,6 ( $\mathrm{n}=51$ ), tipo sanguíneo O positivo 48,1\% (n=38), com média de idade de 51,9 anos, sendo o mais idoso 89 anos e o mais jovem 05 anos. Quanto ao tipo de produto sanguíneo mais utilizado foi o concentrado de hemácias $(\mathrm{CH}) 88,6 \%$ (n=70) sendo $\mathrm{CH}(84,8 \%)$, concentrado de hemácias lavadas (CHL) (1,3\%) e concentrado de hemácias filtradas (CHF) $(2,5 \%)$.

O setor no qual houve maior número de suspeita de RT foi a Unidade de Terapia Intensiva, 21,5\% ( $\mathrm{n}=17$ ), resultado que difere do estudo de Borges, Souza, Masiero e Souza, (2020), que evidenciou a Clínica Médica. Vale destacar que os profissionais que mais notificaram as RT no presente estudo foram os enfermeiros do hospital, $97,5 \%$ (n=77) destes $85,4 \%$ ( $\mathrm{n}=70)$ eram da Agência Transfusional. $\mathrm{O}$ dado corrobora a destacar a necessidade da equipe de enfermagem nos diversos setores que é realizado as transfusões, visto que o enfermeiro é o profissional que monitoriza as fases do processo transfusional e realizou a vigilância do processo de saúde dos pacientes (Conselho Federal de Enfermagem - COFEN, 2016; Frantz et al., 2020; Suddock \& Crookston, 2021), porém, a predominância não era o enfermeiro do setor e sim o enfermeiro que acompanhava o processo transfusional. O hospital possui 94 enfermeiros assistencialista e 6 enfermeiros da agência transfusional.

A instituição abrange unidades de Clínica Médica, Clínica Cirúrgica, Pediatria, Unidade de Terapia Intensiva (UTI), Centro Cirúrgico e Oncologia, atendendo pacientes com comorbidades, que apresentem uma predisposição às RT imediatas. A realização de um screening poderia identificar pacientes vulneráveis e direcionar cuidados que poderiam evitar e/ou reduzir eventos adversos relacionados à transfusão. Também, foram incluídas no estudo as notificações oriundas da UTI COVID, setor de atendimento aos pacientes acometidos por COVID, que foi mantido na instituição, no período de março a setembro de 2020. A UTI COVID gerou (3) três notificações. Salienta-se que não houve um direcionamento quando listado o diagnóstico do paciente ao tipo de RT relacionado (International Society of Blood Transfusion - ISBT, 2018).

Nessa amostra foi evidenciado as neoplasias 32,9\% ( $\mathrm{n}=26)$ associadas a justificativa de maior incidência que foi a Anemia com 57\% $(\mathrm{n}=45)$. Pacientes com neoplasia são predispostos à anemia, maior justificativa para a indicação do HC com potencial de até cinco vezes em relação a pacientes saudáveis, conforme Kenar, Köksoy, Ürün, e Utkan, (2020) ou ainda desencadeado nos pacientes com doença subjacente ou por iatrogenias no transcurso da internação (Ozier, Aubron, \& Nguyen, 2016). Na sequência é apresentada (Tabela 1), com a relação de classificação de reações transfusionais: 
Tabela 1 - Distribuição das frequências das notificações de reações transfusionais, Aracaju, SE - Brasil, 2017 a 2020.

\begin{tabular}{ll}
\hline Diagnóstico & $\mathbf{N}(\%)$ \\
\hline Suspeita de Reações transfusionais & \\
TACO & $14(17,7)$ \\
RFNH & $44(55,7)$ \\
RALG & $14(17,7)$ \\
TRALI & $07(8,9)$ \\
DRT & $02(2,5)$ \\
CB & $01(1,3)$ \\
TAD & $02(2,5)$ \\
\hline
\end{tabular}

Nota: TACO: Sobrecarga Volêmica Associada à Transfusão; RFNH: Reação Febril não Hemolítica; RALG: Reação Alérgica; TRALI: Lesão Pulmonar Relacionada à Transfusão; DRT: Dor Relacionada a Transfusão; CB: Contaminação Bacteriana; TAD: Dispneia associada à Transfusão. Fonte: Autores (2021).

A classificação do incidente foi considerada pelos notificadores como sem danos em $66 \%$ (n=52) em comparação aos pacientes elencados como com dano e extensão da hospitalização em $23 \%$ (n=18) e não souberam informar com 11\% (n=09). O óbito pós transfusional foi evidente em 2,5\% $(\mathrm{n}=2)$, sendo prontamente notificado no prazo de $72 \mathrm{~h}$ do ocorrido à Agência de Vigilância Sanitária (ANVISA, 2015). A gravidade foi evidenciada da seguinte forma conforme a estratificação no SCIRAS: leve $84,8 \%(n=67)$, moderado $11,4 \%(n=09)$, grave $1,3 \%(n=01)$, e óbito 2,5\% $(n=2)$, Na amostra, todos os eventos leves foram caracterizados tanto pela RFNH (55,7\%) quanto pela RALG $(17,7 \%)$, esses dados divergem de um estudo realizado em 2018 (Grandi, Grell, Areco, \& Barbosa, 2018). Os profissionais apresentaram uma estimativa demasiada em relação ao dano causado pela suspeita de uma RT, porém, essa inferência sobre a perspectiva do notificador quanto à suspeita, não foi localizada na literatura científica.

No processo transfusional as plaquetas devem ser infundidas no mínimo de 30 minutos a no máximo de 60 minutos (Agência Nacional de Vigilância Sanitária - ANVISA, 2015). O prazo mínimo correspondeu a 29\% (02) e o prazo máximo foi de 57\%, (04) apenas 14\% \% (01) representou o tempo ideal, conforme os manuais e Procedimento Operacional Padrão brasileiros (Empresa Brasileira de Serviços Hospitalares - Ebserh, 2021). Por outro lado, a análise do tempo de infusão preconizado para o concentrado de hemácias se concretizou de acordo com a literatura vigente com apenas $1 \%$ (01) com prazo extrapolado. A mediana distribuiu dessa forma: CH - 120 minutos; CHF - 175 minutos, CHL - 100 minutos, PFC - 15 minutos, Plaquetas - 120 minutos. Na sequência, o tempo de infusão do HC e demonstrado através do Gráfico 1: 
Gráfico 1 - Tempo de infusão dos HC, Aracaju, SE - Brasil, 2017 a 2020.

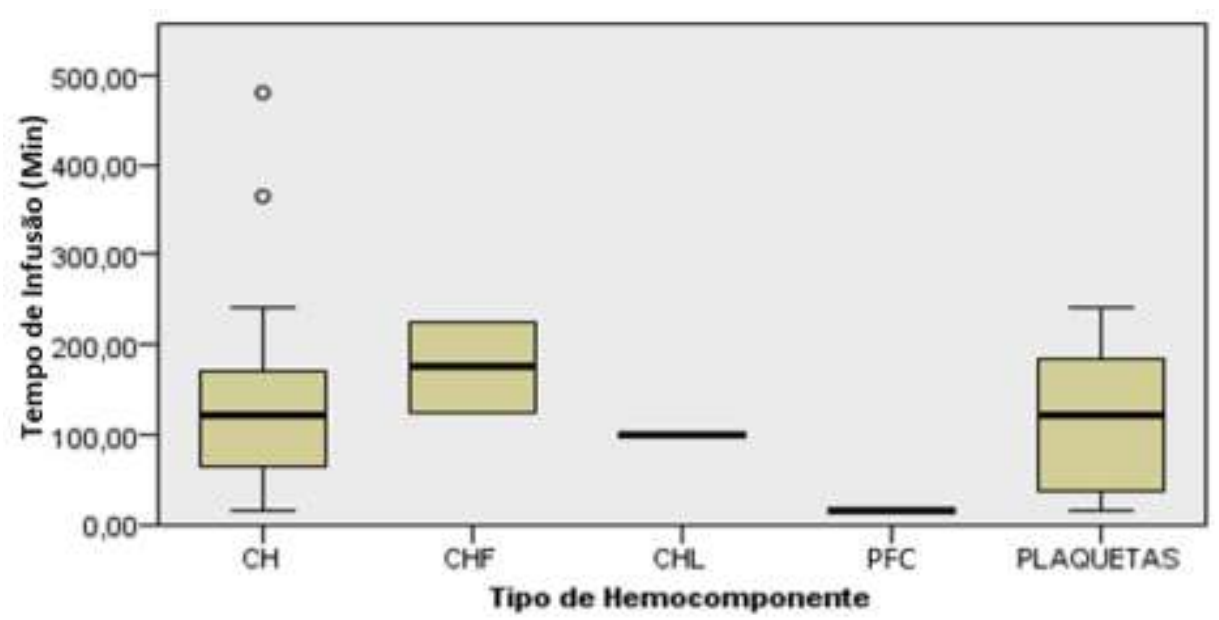

Nota: CH: Concentrado de Hemácias; CHF: Concentrado de Hemácias Filtradas; CHL: Concentrado de Hemácias Lavadas; PFC: Plasma Fresco Congelado. Fonte: Autores (2021).

Entre os sinais e sintomas voltados para as RT conforme CDC/NHSN (2018/2020), buscou-se, nessa pesquisa, associar os exames solicitados, os resultados, o tratamento efetivado, o recolhimento das amostras da bolsa do HC e do paciente, em suma, as condutas adotadas pela equipe quanto à suspeita diagnóstica à beira leito. Uma vez que essa sequência de atividades definirão a causalidade de uma RT. Dessa forma, a notificação ainda que seja realizada a posteriori se as condutas dos profissionais foram pertinentes e a detecção foi realizada (com elementos clínicos, laboratoriais e terapêuticos) no período do lapso temporal das RT servirão como comprobatórias para a RT. Destacou-se os seguintes sinais e sintomas, de acordo com a Tabela 2:

Tabela 2 - Manifestações clínicas associadas a RT, Aracaju, SE - Brasil, 2017 a 2020.

\begin{tabular}{ll}
\hline Sinais e Sintomas & $\mathbf{N}(\%)$ \\
\hline Calafrio & $19(24,1)$ \\
Febre & $49(62,0)$ \\
Urticária & $09(11,4)$ \\
Prurido & $06(7,6)$ \\
Dispneia & $12(15,2)$ \\
Dor em MMII & $01(1,3)$ \\
Dor abdominal & $01(1,3)$ \\
Dor torácica & $04(5,1)$ \\
Hipotensão & $01(1,3)$ \\
Náuseas/Vômitos & $03(3,8)$ \\
Rash cutâneo & $06(7,6)$ \\
Cefaleia & $02(2,5)$ \\
\hline
\end{tabular}

Nota: MMII: Membros Inferiores. Fonte: Autores (2021).

Nessa amostra, a possibilidade de RT levantadas a partir do sistema de notificações informatizado foi: RFNH 55,7\% $(\mathrm{n}=44)$, RALG 17,7\% (n=14) com igualdade com a TACO, RFH (0), TRALLI 8,9\% (n=07), Dor relacionada à Transfusão (DRT) 2,5\% (02) e TAD também com resultado similar. Diante dessas suspeitas de RT, os exames quando solicitados configuraram-se predominantemente de forma parcial não descartando de forma ampla outras RT sendo o recordista o hemograma com 32,9\%. Diante de reações cardiopulmonares, o raio x representou apenas em 2,5\% diante de uma amostra de 29,1\% e nenhuma hemogasometria relatada. Em relação aos exames realizados e do tratamento ofertado foram realizadas considerações a seguir a partir das especificidades de cada RT. O tipo sanguíneo com maior índice para a RT foi o “O” com 
RH positivo para RFNH (51,2\%), TACO (46,2\%), RALG (58,3\%), TRALI (66,7\%), DRT (50\%) e TAD (50\%). Na CB (n=1) com $100 \%$ para B positivo.

Quanto aos registros que não contemplaram a informação acerca dos exames (39,2\%), denota-se que a intenção só foi de notificar. Nenhum paciente foi contemplado com exames direcionados para a suspeita de RT de acordo com as recomendações propiciando o potencial de recidivas e custos descabidos. Isso se comprova com exames variados (mesclados) ou de forma genérica sem discriminar os exames e até sem relação alguma com a RT (Tabela 3):

Tabela 3 - Exames classificados quanto à solicitação no hospital diante da suspeita de RT, Aracaju, SE - Brasil, 2017 a 2020.

\begin{tabular}{ll}
\hline Exames & $\mathbf{N}(\mathbf{\%})$ \\
\hline Não solicitado & $05(6,3)$ \\
Sem relato & $31(39,2)$ \\
Parcial & $21(26,6)$ \\
Sem especificar (sem discriminar) & $06(7,6)$ \\
Variado (mescla de solicitação diversas) & $06(7,6)$ \\
Sem relação com a suspeita de RT & $10(12,7)$ \\
\hline Hemograma & $26(32,9)$ \\
Radiografia de tórax & $02(2,5)$ \\
Hemogasometria & $00(0,0)$ \\
\hline
\end{tabular}

Fonte: Autores (2021).

As notificações sem relatos de exames para cada RT estão discriminadas conforme a suposição de RT, o que pode ter denotado que os sinais e sintomas já seriam suficientes para confirmar na percepção dos profissionais uma RT. Na sequência, a amostra $(n=33)$ de notificações estudadas com RT e registro de exames solicitados, foram classificados de acordo com a suspeita de RT (Gráfico 2):

Gráfico 2 - Exames solicitados diante da suspeita de RT, Aracaju, SE - Brasil, 2017 a 2020.

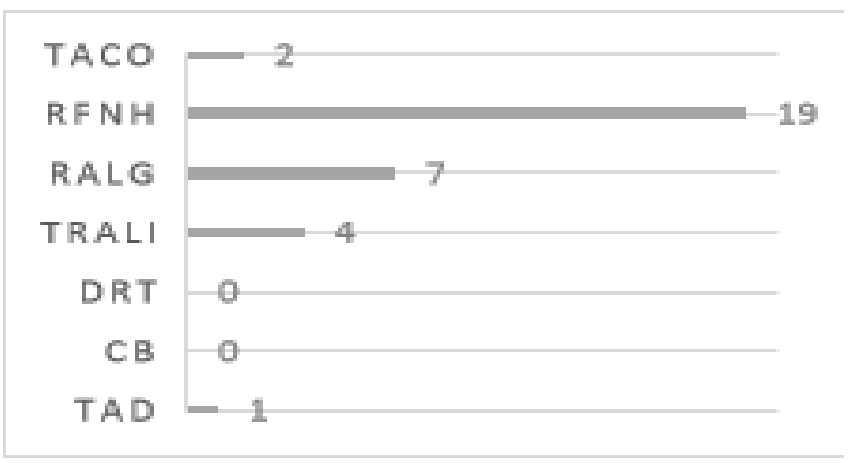

Nota: TACO: Sobrecarga Volêmica Associada à Transfusão; RFNH: Reação Febril não Hemolítica; RALG: Reação Alérgica; TRALI: Lesão Pulmonar Relacionada à Transfusão; DRT: Dor Relacionada a Transfusão; CB: Contaminação Bacteriana; TAD: Dispneia associada à Transfusão. Fonte: Autores (2021).

Quanto ao tratamento, foi avaliado se houve efetividade para a especificidade da RT. Observou-se que o uso de anti térmicos para RFNH (76,9\%), TACO (7,7\%), TRALI (11,5\%), DRT (3,8\%) e CB (3,8\%); anti histamínico: RALG (83,3\%) e TRALI (16,7\%); anti hipertensivo TACO (100\%); diurético TACO (66,7\%) e TAD (33,3\%); corticóide TACO (33,3\%), RFNH (16,7\%), TRALI (66,7\%) e TAD (16,7\%); anti emético: RFNH (50\%) e CB (50\%). Sem a especificação do tratamento distribuiu-se da seguinte maneira: TACO (20\%), RFNH (20\%), RALG (40\%), DRT (20\%).

Para a reação alérgica (RALG) oriundas de RT, outros elementos são destacados como possíveis desencadeadores de eventos transfusionais, como: efeito de medicamentos, de alimentos e outros alérgenos. Nesse estudo, a urticária foi o sintoma 
mais relevante, porém, sem um segundo elemento causador RT, não confere como RALG definitiva, conforme o NHSN (2018). Como faz-se necessário dois elementos dos sinais e sintomas para caracterizar RALG, só teria como suspeitar em 23,07\% da amostra estudada. Foi a segunda RT de rápida iniciação das manifestações clínicas assim como foi também a que houve rápida notificação (no mesmo dia do evento) denotando a rápida assimilação do profissional com a suspeita de RT. A mediana apresentou-se da seguinte maneira: TAD: 15 minutos, TRALI: 75 minutos, RALG: 70 minutos, TACO: 110 minutos, RFNH: 140 minutos, DRT: 145 minutos. Na sequência, foi mostrada a distribuição do início das manifestações clínicas das RT através do (Gráfico 3):

Gráfico 3 - Distribuição do início das manifestações clínicas das RT, Aracaju, SE - Brasil, 2017 a 2020.

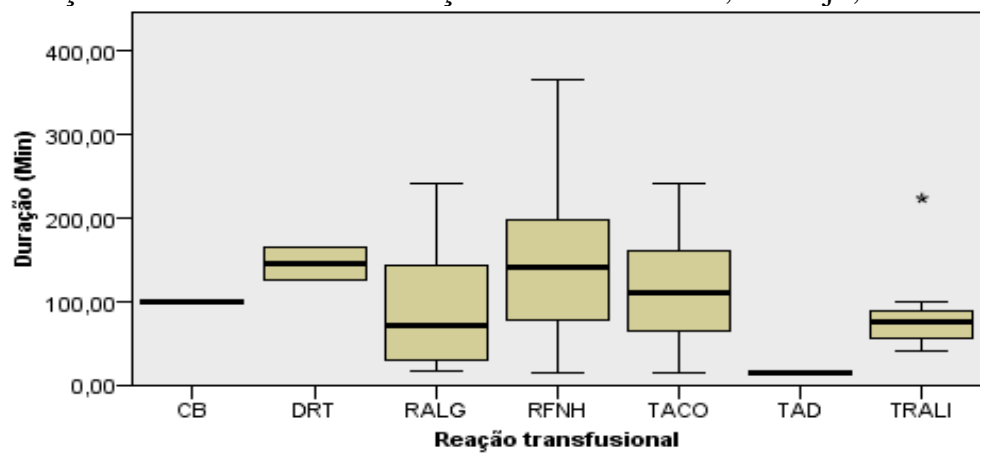

Nota: CB: Contaminação Bacteriana; DRT: Dor Relacionada a Transfusão; RALG: Reação Alérgica; RFNH: Reação Febril não Hemolítica; TACO: Sobrecarga Volêmica Associada à Transfusão; TAD: Dispnéia associada à Transfusão; TRALI: Lesão Pulmonar Relacionada à Transfusão. Fonte: Autores (2021).

Percebeu-se que não foi solicitado exames em 53,8\% das situações em que se deve focar nos sinais e sintomas como o preconizado pela CDC/DHSN $(2018,2021)$. No entanto, a coleta da amostra do paciente e da bolsa do HC não englobam como comprovação para RALG, embora seja uma conduta adotada pelo Ministério da Saúde diante de qualquer suspeita de RT, em 28,5\% 9 (Agência Nacional de Vigilância Sanitária - ANVISA, 2015). Os exames que poderiam ser voltados para determinar RALG seriam a dosagem de IgE e eosinófilos no campo da imunologia (Souza et al., 2019), mas confirmação busca apenas elementos clínicos diante dos protocolos da CDC/NHSN, (2018, 2021).

Em contrapartida, como todas as manifestações associadas a RALG nessa pesquisa foram classificadas como leves, os HC foram interrompidos e deveriam ser reinstalados conforme preconizado IPST (2016). Estudos apontam que esses pacientes poderão ser hemotransfundidos novamente em $48 \mathrm{~h}$ (Kasim, Aldarweesh, \& Connor, 2019) podendo até predispor a casos de aloimunizações futuras (Adkins, Lawicki, Johnson, \& Eichbaum, 2019). Nessa pesquisa, nenhuma transfusão foi tentada novamente após a interrupção da infusão e a resolução do quadro clínico. Apenas 14,2\% (n=01) foi concluída (seguiu o transcurso) provavelmente sem suspeição de RT porque não houve interrupção descrita.

Em associação ao tratamento de RALG voltado apenas para sintomas localizados e cutâneos engloba a administração de anti-histamínico. Nessa amostra, todos deveriam receber apenas esse tratamento, porém receberam outras linhas medicamentosas. Embora na literatura vigente o recomendado para a administração de epinefrina, anti histamínico, broncodilatadores e corticoides seria para as reações anafiláticas (Goel, Tobian, \& Shaz, 2019). Para RALG de menor proporção, deve ser recomendado anti histamínico e/ou corticóide já que outra terapêutica como anti térmico não condiz com essa RT (Serious Hazards of Transfusion - SHOT, 2019).

$\mathrm{Na}$ forma grave, pacientes podem ser testados para deficiência críticas de $\operatorname{IgA}$ e assim utilizar HC oriundos de doadores portadores deficiência de IgA ou lavados (Simons et al., 2015). Na próxima transfusão, já deve ser indicado o HC lavado (NHSN - National Healthcare Safety Network, 2021). 
No que concerne a RFNH, esse estudo, confirmou sua predominância como RT, assim como outros resultados na literatura (Bueno, Milani, \& Soares, 2019). Nessa RT, a febre é o sinal prevalente de todas as notificações com uma taxa de $62 \%$ e com uma média da temperatura de inicial de $36,4^{\circ} \mathrm{C}$ a temperatura final de $38,3^{\circ} \mathrm{C}$. É caracterizada por febre ao menos $1^{\circ} \mathrm{C}$ em relação ao valor pé transfusional associado ou não a calafrios, tremores, cefaleia, náuseas e vômitos (Agência Nacional de Vigilância Sanitária - ANVISA, 2015). Ocorre durante ou até 4 horas após a cessação da transfusão sem outras causas que expliquem os sinais e sintomas (Veiga \& Rojas, 2018).

No entanto, para diferenciar a RFNH de Contaminação Bacteriana (CB) entre outros elementos, faz-se necessário a hemocultura do paciente e da amostra da bolsa do HC para constatar a presença de patógenos entre as amostras e sem nenhum outro risco potencial para a infecção desse patógeno prévio à transfusão (CDC/DHSN, 2021). As hemoculturas quando pedidas seguiram de forma ideal (amostra do paciente e da bolsa do HC) em 19,2 \% em detrimento das que foram solicitadas apenas de uma amostra (paciente ou da bolsa do HC) em 12,8\% e que não descarta a RT.

Ainda sobre essa abordagem, acrescenta-se que mesmo sem febre foi solicitado hemocultura das duas amostras (paciente e bolsa) ou apenas uma amostra, representando falha de processo, visto que o exame solicitado não justifica necessariamente a condição clínica de um paciente, representando erro de conduta e não sequenciamento dos protocolos institucionais ou protocolos internacionais sobre RT, gerando desperdício e gasto supérfluos, tal relação verifica-se na (Tabela 4):

Tabela 4 - Solicitação de hemoculturas de pacientes e das bolsas do HC na vigência da Febre, Aracaju, SE - Brasil, 2017 a 2020.

\begin{tabular}{lllll}
\hline \multirow{2}{*}{ Coleta } & \multicolumn{2}{c}{ Febre } & \multirow{2}{*}{ Total } & \multirow{2}{*}{$\mathbf{p}^{\mathbf{a}}$} \\
\cline { 2 - 3 } & \multicolumn{1}{c}{ Não } & Sim & \\
\hline Coleta de amostras (paciente e da bolsa do HC) & $35(71,4)$ & $63(80,8)$ & \multirow{2}{*}{0,007} \\
Não & $28(96,6)$ & $14(28,6)$ & $15(19,2)$ & \\
Sim & $1(3,4)$ & & & \\
\hline Coleta de uma amostra & & $42(85,7)$ & $68(87,2)$ & 0,736 \\
Não & $26(89,7)$ & $7(14,3)$ & $10(12,8)$ & \\
Sim & $3(10,3)$ & &
\end{tabular}

Nota: Amostras coletadas, não tiveram repetições entre si; $\mathbf{p}^{\mathbf{a}}$ - Teste Exato de Fisher; $\mathbf{p}<0,05$. Fonte: Autores (2021).

Sobre os exames subsidiários da RFNH, não foi observado nenhum artigo da literatura sobre exames específicos uma vez que a RFNH é diagnóstica de exclusão para Lesão Pulmonar relacionado à Transfusão (TRALI), Contaminação Bacteriana (CB) e Reação Febril Hemolítica - RFH (Veiga \& Rojas, 2018). Não obstante, para Goel, Tobian e Chaz (2019), deve-se rever a identificação da bolsa e do paciente, certificar-se do sistema $\mathrm{ABO}$ e proceder com o teste direto de antiglobulina (TDA). Ressalta-se que a dosagem de IgA deve estar atrelada a RALG e não a RFNH apesar de ter sido realizado em 1\% da amostra.

Sabe-se que TACO e TRALI estão dentre os eventos adversos transfusionais mais subnotificado no mundo não sendo distinto nesse estudo. Todos os pacientes que evoluíram com dispneia ainda que não sejam portadores de comorbidades conforme o IBST (2018) ou sem quadro clínico contundente dessas reações, deveriam ser triados e realizado a coleta de hemogasometria e efetivado a radiografia de tórax no período temporal adequado para solidificar o diagnóstico e proceder a prevenção.

Todavia, apenas dois pacientes foram beneficiados com a realização de apenas a radiografia de tórax o que amplifica a subnotificação nesse estudo para possivelmente em $29,1 \%$ dos casos. O tratamento fornecido para esses pacientes envolveu diuréticos e anti hipertensivos voltados para TACO e para TRALI não há tratamento específico apenas suporte ventilatório. Em consoante, o uso de corticoides e anti histamínicos não englobam o tratamento específico para essas RT (Reese, 2017). 
Ainda sobre TACO, liderou no aspecto interrupção da infusão com 42,9\%. Para pacientes com sobrecarga volêmica o peso, a altura e a velocidade de infusão podem ser determinantes para essa RT. A administração do HC precisa ser de forma cuidadosa, não deve ultrapassar uma infusão $2 \mathrm{ml} / \min$ ou $2 \mathrm{ml} / \mathrm{kg} / \mathrm{h}$ em pacientes de alto risco e não poderá ser administrado de forma simultânea (Aubron, Aries, Le Niger, Sparrow, \& Ozier, 2018). Com isso, foi verificado a inobservância pelo profissional que acompanha o ato transfusional ou daquele que libera um HC com um volume inadequado para o índice de massa corporal (IMC) e, como deve ser infundido no tempo preconizado, pode caracterizar a sobrecarga cardíaca em pacientes com fatores predisponentes.

Em relação a suspeição das RT, nos EUA, o protocolo do CDC/NHSN (2020) exibe um impresso próprio que já direciona os aspectos assistenciais para a especificidade da RT e aborda outras variáveis como o tipo sanguíneo, idade, cor, sexo, tipo do HC, histórico transfusional, gravidade, imputabilidade, tratamento e desfecho. E, assim conduz de forma eloquente e assertiva a conduta apropriada. Assim, a prevenção será alcançada após o diagnóstico da RT firmado.

Tendo visto a conduta dos profissionais envolvidos, partiu-se para a assimilação do lapso temporal diante da notificação concretizada. Entre a data do incidente transfusional e a notificação no sistema conferiu um tempo médio de 3,91 (aproximadamente 04 dias) com uma mediana abaixo de 03 dias para os profissionais notificarem. No entanto, se os exames fossem realizados corretamente poderiam ser correlacionados de forma célere ainda que o envio desses dados à ANVISA para a conclusão da investigação seja tardio como ocorreu com uma média de 32 dias nesse estudo (Tabela 5):

Tabela 5 - Tempo entre a suspeita de RT, a abertura e a conclusão da investigação, Aracaju, SE - Brasil, 2017 a 2020.

\begin{tabular}{llllll}
\hline \multirow{2}{*}{ RT } & \multicolumn{4}{c}{ Termo de Tendência Central e Amplitude } & \multirow{2}{*}{$\mathbf{p}^{\mathbf{a}}$} \\
\cline { 2 - 5 } & Mediana & Intervalo interquartil & Mínimo & Máximo & 0,752 \\
DRT & 1,5 & - & 0 & 3 & \\
RALG & 1 & 4 & 0 & 10 & \\
RFNH & 1 & 6 & 0 & 314 & \\
TACO & 1,5 & 6,25 & 0 & 80 & \\
TAD & 3 & - & 2 & 4 & \\
TRALI & 0 & 1 & 0 & 6 & \\
\hline
\end{tabular}

Nota: Ressalva sobre a Tabela 5, composta do tempo entre a suspeita de RT, notificação, investigação e conclusão em dias; $\mathbf{p}^{\mathbf{a}}$ - Teste Kruskal-Wallis. Fonte: Autores (2021).

Outro aspecto verificado foi a possibilidade de recidiva. Assim, o histórico transfusional não foi preenchido em $85 \%$ (67) das notificações. A possibilidade de recidivas de RT nesses pacientes ocorreu entre 01 dia a 18 dias do evento inicial e apenas $27 \%(\mathrm{n}=3)$ estavam em unidades distintas, ou seja, a possibilidade de recidiva poderia ter sido identificada e medidas preventivas adotadas de forma intersetorial. Além disso, demonstrou-se que a ausência dessas informações pertinentes ao histórico transfusional não foi repassada pelos os profissionais de saúde tanto em setores distintos como nos mesmos locais de lotação (Tabela 6). Vale salientar que o paciente pode apresentar outras RT, simultaneamente (Kathryn E. Webert \& Nancy M. Heddle, 2017): 
Tabela 6 - Potencial de recidiva dos pacientes, Aracaju, SE - Brasil, 2017 a 2020.

\begin{tabular}{ll}
\hline Variável & $\mathbf{N}(\%)$ \\
\hline Paciente apresentou recidiva & \\
Sim & $11(15,9)$ \\
Não & $58(84,1)$ \\
\hline Os reincidentes estavam no mesmo setor & \\
Sim & $8(72,7)$ \\
Não & $3(27,3)$ \\
\hline As reincidências eram as mesmas RT & \\
Sim & $5(45,5)$ \\
Não & $6(54,5)$ \\
\hline
\end{tabular}

Fonte: Autores (2021).

Foi realizada a análise do sistema VIGIHOSP com a Ficha de notificação FIT. Verificou-se que o sistema informatizado da instituição só foi eficaz no ano de 2019 e de 2020 uma vez que iniciou de forma desapercebida nos anos de 2016, 2017 e 2018. Em 2019 e 2020, com treinamentos fornecidos pela instituição, percebeu-se que as notificações via sistema apresentaram uma combinação perfeita (match) com as FIT. No entanto, as FIT contabilizaram repetições no ano de 2019 $(n=11)$ em contraste com o sistema de notificação que os excedentes foram notificados sem repetições tanto para 2019 quanto para 2020.

As flutuações de ausência de notificação nesse período se comportaram da mesma forma como o processo foi estreado, ou seja, a FIT como padrão ouro nos anos 2016 a 2018 em relação ao sistema de notificação. O sistema de notificação pelo aplicativo foi mais acionado no ano de 2019, em contrapartida, no ano de 2020 as notificações tanto pelo VIGIHOSP como pela FIT mantiveram-se compatíveis nos mesmos meses.

Ademais, o estudo obteve uma amostra robusta. Em 2020, as RT confirmadas ficaram acima do previsto para o sistema brasileiro (05 RT/1000 transfusões) totalizando 07 RT somente nessa instituição em 2020 com 1081 transfusões (sem mencionar Sergipe/ Brasil) e distante da meta do sistema francês (03 RT/1000 transfusões) sugerindo potencial de subnotificação nos anos anteriores.

Vale ressaltar que os casos de subnotificação são derivados de: da falta de conhecimento sobre a temática; da redução da assimilação de relacionar as manifestações clínicas com a possibilidade de RT; perda do lapso temporal; do tratamento que teoricamente ocorrerá sem essa correlação e assim passará imperceptível esse evento adverso, principalmente, para a TACO e TRALI; sintomas em pacientes inconscientes ou comatosos não serão percebidos (apenas sinais) e condições do cliente prévias como o quadro do paciente crítico podem descaracterizar ou não concretizar a imputabilidade confirmada (Sahu \& Bajpai, 2020).

Neste estudo, a incompletude das informações tanto dos exames laboratoriais e de imagem solicitados, das amostras (paciente e da bolsa do HC), do lapso temporal e das manifestações clínicas que dificultaram a análise e a confirmação da RT. Segundo Gurgel et al. (2019) deve-se enfatizar a necessidade de registros completos e criar estratégias para mitigar a ausência de informações como observada por Soares, Cruz, Almeida, Silva Camilo e Scopacasa (2020). Por conseguinte, sem a definição de uma RT, as medidas de prevenção não serão efetivadas. Enfatiza-se que dessas suspeitas diagnósticas (n=79) apenas onze terem sido confirmadas após a elaboração da FIT diante de exames comprobatórios.

\section{Considerações Finais}

As limitações do estudo foram o "N" da amostra estudada que poderia mostrar outros resultados e discussões, ao mesmo tempo que o "N" da amostra está relacionado diretamente com o problema que é investigado, na tentativa de propor soluções para mitigar o problema da subnotificação nas Reações Transfusionais. A observação de lacunas na literatura 
especializada sobre o tema estudado que não permitiu aprofundar a discussão de alguns resultados apresentados neste texto. Outra dificuldade apontada foi a possibilidade de subnotificação de reações transfusionais imediatas no período assinalado pela vigência da COVID em consequência da dependência da confecção de FIT pelos médicos para prosseguir com a investigação.

A amostra incluída neste estudo mostrou que os exames quando solicitados não foram direcionados nem para a suspeita diagnóstica nem para o tratamento ofertado, o que acarretou desperdício com tratamento aos pacientes transfundidos, ainda o tratamento dispensável para o paciente o expõe a situações não seguras do processo transfusional além da exposição ao ambiente hospitalar e ambientes com patógenos diversos.

As reações transfusionais quando notificadas demonstraram uma fonte rica para um cuidado mais adequado para pacientes submetidos ao tratamento transfusional com hemocomponentes. Isso pode ser verificado pela caracterização da heterogenia das RT, segundo preenchimento das notificações que variou dentro da amostra estudada. E, considerando essa variação pode-se considerar uma diversidade de RT e as classificações segundo dos manuais nacionais e internacionais, problemas iatrogênicos, além de comprometer o planejamento do cuidado da equipe multiprofissional.

As contribuições para área podem ser destacadas, quanto a riqueza de referências relacionadas aos parâmetros apresentados dos diversos manuais internacionais usados para classificar as diversas reações transfusionais. Ainda, destaca-se que esse manuscrito pretende compor o corpo de conhecimento científico para futuros estudos sobre o tema investigado. As variáveis investigadas neste estudo se mostraram relevantes, porém estudos sequenciais com outros delineamentos metodológicos e com o uso de dados primários, podem reforçar estas evidências e são recomendados.

\section{Agradecimentos}

Os autores gostariam de agradecer ao setor de Serviço de Controle de Infecções Relacionadas à Assistência à Saúde em especial a Milena Xavier, a Agência de Vigilância Sanitária em especial a Gilvan Barreto, a Agência Transfusional em especial a Dr. Geidson Cruz que foram de extrema importância para a realização deste estudo.

\section{Referências}

Adkins, B. D., Lawicki, S., Johnson, M., \& Eichbaum, Q. (2019). Mild Allergic Transfusion Reactions. American Journal of Clinical Pathology, 151(3), 344348. https://doi.org/10.1093/ajcp/aqy150

Agência Nacional de Vigilância Sanitária - ANVISA. (2015). Marco Conceitual e Operacional de Hemovigilância: Guia para a Hemovigilância no Brasil. Brasília, Brasil.

Agência Nacional de Vigilância Sanitária - ANVISA. (2016). Hemovigilância no Brasil - Relatório consolidado 2007 - 2015. Brasilia. Retrieved from https://www.gov.br/anvisa/pt-br/assuntos/fiscalizacao-e-monitoramento/hemovigilancia/publicacoes/hemovigilancia-no-brasil-relatorio-consolidado-20072015.pdf/view

Agence nationale de sécurité du medicament - ANSM. (2018). Sécurité du Médicament et des produits de Santé. ANSM. L'ANSM publie le rapport d'activité hémovigilance 2018. Retrieved from https://ansm.sante.fr/qui-sommes-nous/publications-institutionnelles/publications-2019

Aubron, C., Aries, P., Le Niger, C., Sparrow, R. L., \& Ozier, Y. (2018). How clinicians can minimize transfusion-related adverse events? Transfusion Clinique et Biologique, 25(4), 257-261. https://doi.org/10.1016/j.tracli.2018.08.158

Borges, K. V., Souza, M. A., Masiero, A. V., \& Souza, P. A. (2020). Análise da Eficácia Transfusional através dos Exames Pré e Pós Transfusionais Em Idosos no Hospital Tereza Ramos em Lages, Santa Catarina. Hematology, Transfusion and Cell Therapy, 42(S2), 371. https://doi.org/10.1016/j.htct.2020.10.624

Bosboom, J. J., Klanderman, R. B., Peters, A. L., van de Weerdt, E. K., Goudswaard, E. J., Binnekade, J. M., \& Vlaar, A. P. (2018). The practice of diagnosing and reporting transfusion-associated circulatory overload: a national survey among physicians and haemovigilance officers. Transfusion Medicine, 28(5), 363-370. https://doi.org/10.1111/tme.12480

Brasil. Resolução no 466, de 12 de dezembro de 2012. Dispõe sobre diretrizes e normas regulamentadoras de pesquisas envolvendo seres humanos. , Diário Oficial da União § (2012). BRASIL.

Bueno, C. S., Milani, C. L. de S., \& Soares, S. C. de L. (2019). Epidemiologia das reações tranfusionais imediatas notificadas em um hospital de alta complexidade no interior de Rondônia. Revista Recien - Revista Científica de Enfermagem, 9(25), 77. https://doi.org/10.24276/rrecien23583088.2019.9.25.77-84 
Canadian Blood Services - CBS. (2017). System Progress Reports. https://www.blood.ca/en/about-us/publications-and-reports/system-progress-reports.

Center for Disease Control/National Healthcare Safety Network - CDC/NHSN. (2018). NHSN Biovigilance Component, Hemovigilance Module Protocol. The United States of America. Retrieved from https://www.cdc.gov/nhsn/acute-care-hospital/bio-hemo/index.html.

Center for Disease Control/National Healthcare Safety Network - CDC/NHSN. (2021). National Healthcare Safety Network biovigilance component hemovigilance module surveillance Protocol v2.6. The United States of America. Retrieved from https://www.cdc.gov/nhsn/pdfs/biovigilance/bv-hv-protocolcurrent.pdf.

Coberly, E. A., \& Booth, G. S. (2016, February 1). Ten-year retrospective review of transfusion practices in beating-heart organ donors. Transfusion, 56, 339343. Blackwell Publishing Inc. https://doi.org/10.1111/trf.13340.

Conselho Federal de Enfermagem - COFEN. Resolução Cofen n ${ }^{\circ} 511$ de 29 de março de 2016 Conselho Federal de Enfermagem - Brasil. , Diário Oficial da União $§(2016)$.

Empresa Brasileira de Serviços Hospitalares - Ebserh. (2021). Procedimento Operacional Padrão Código: POP ENF 15.1 Rotina de Instalação de Hemocomponentes para Transfusão. Rio de Janeiro.

Frantz, S. R. de S., Vargas, M. A. de O., Pires, D. E. P. de, Brito, M. J. M., Bitencourt, J. V. de O. V., \& Ribeiro, G. (2020). Nursing work and competence in hemotherapy services: an ergological approach. Revista Brasileira de Enfermagem, 73(3), e20180775. https://doi.org/10.1590/0034-7167-2018-0775.

Gehrie, E. A., Hendrickson, J. E., \& Tormey, C. A. (2015). Measuring the influence of blood component infusion rate on recipient vital signs. Vox Sanguinis, 109(4), 353-358. https://doi.org/10.1111/vox.12310.

Goel, R., Tobian, A. A. R., \& Shaz, B. H. (2019). Noninfectious transfusion-associated adverse events and their mitigation strategies. Blood, 133(17), 18311839. https://doi.org/10.1182/blood-2018-10-833988.

Grandi, J. L., Grell, M. C., Areco, K. C. N., \& Barbosa, D. A. (2018). Hemovigilância: a experiência da notificação de reações transfusionais em Hospital Universitário. Revista Da Escola de Enfermagem Da USP, 52, e03331. https://doi.org/10.1590/s1980-220x2017010603331.

Gurgel, A. P., Melo, V. S. de, Leitão, J. S., Studart, R. M. B., Bonfim, I. M., \& Barbosa, I. V. (2019). Paciente crítico: segurança em terapia transfusional mediante lista de verificações. Revista Brasileira de Ciências Da Saúde, 23(4), 525-534. https://doi.org/10.22478/ufpb.2317-6032.2019v23n4.37205

Hinkle, D. E., Wiersma, W., \& Jurs, S. G. (2003). Applied statistics for the behavioral sciences (Vol. 663). Houghton Mifflin College Division.

Instituto Português do Sangue e da Transplantação - IPST. (2016). Instituto Português do Sangue $e$ da Transplantação. https://www.ine.pt/xportal/xmain?xpid=INE\&xpgid=ine_indicadores\&indOcorrCod=0006132\&contexto=bd\&selTab=tab2.

Instituto Português do Sangue e da Transplantação - IPST. (2019). Algoritmos de notificação o de Reação es Adversas em Receptores Sistema Português de Hemovigilância. http://ipst.pt/index.php/pt/dados-estatisticos-transplantacao.

International Society of Blood Transfusion/International Haemovigilance Network, ISBT/IHN. (2019). Definição revisada de caso de vigilância internacional de sobrecarga circulatória associada à transfusão: uma classificação estudo de validação de contrato. https://www.isbtweb.org/

Kasim, J., Aldarweesh, F., \& Connor, J. P. (2019). Blood product and laboratory resource wastage in non-severe allergic transfusion reactions: an opportunity for improvement. Transfusion Medicine, 29(5), 338-343. https://doi.org/10.1111/tme.12630.

Kathryn E. Webert, \& Nancy M. Heddle. (2017). Investigation of Acute Transfusion Reactions. In M. F. Murphy \& D. H. Pamphilon (Eds.), Practical Transfusion Medicine (5th ed., pp. 69-80). Oxford, UK: Blackwell Publishing Ltd. http://doi.wiley.com/10.1002/9780470988411.

Kenar, G., Köksoy, E. B., Ürün, Y., \& Utkan, G. (2020). Prevalence, etiology and risk factors of anemia in patients with newly diagnosed cancer. Supportive Care in Cancer, 28(11), 5235-5242. https://doi.org/10.1007/s00520-020-05336-w.

Kim, J., \& Na, S. (2015). Transfusion-related acute lung injury; clinical perspectives. Korean Journal of Anesthesiology, 68(2), 101. https://doi.org/10.4097/kjae.2015.68.2.101

Lee, S. J., Wang, H., Ahn, S. H., Son, M. K., Hyun, G. H., Yoon, S. J., \& Kwon, S. W. (2019). Metabolomics Approach Based on Multivariate Techniques for Blood Transfusion Reactions. Scientific Reports, 9(1), 1740. https://doi.org/10.1038/s41598-018-37468-9.

Marconi, M., \& Lakatos, E. (2003). Fundamentos de metodologia científica. In Editora Atlas S. A. (6th ed.). https://doi.org/10.1590/S151797022003000100005

Nazário, S. da S., Barancelli, M. D. C., Gandolfi, M., Marcondes, C., \& Lilian Moura de Lima Spagnolo. (2019). Educação permanente de equipe de enfermagem em reação transfusional. Rev. Enferm. UFPE on Line, 13(2), 307-314. https://doi.org/10.5205/1981-8963-v13i2a237361p307-314-2019.

National Healthcare Safety Network - NHSN. (2021). National Healthcare Safety Network biovigilance component hemovigilance module surveillance Protocol. Atlanta. https://www.cdc.gov/nhsn/pdfs/biovigilance/bv-hv-protocol-current.pdf.

Ozier, Y., Aubron, C., \& Nguyen, B.-V. (2016). Anémies nosocomiales : réalité, conséquences et prévention. Transfusion Clinique et Biologique, 23(4), 185191. https://doi.org/10.1016/j.tracli.2016.07.005.

Reese, F. B. (2017). Plasma e Concentrado de Complexo Protrombínico. In PROAMI Programa de Atualização em Medicina Intensiva (1st ed., Vol. 1, pp. 97145.). Alegre: Artmed Panamericana.

Ribeiro, W. A., Faillace, G. B. D., Fassarella, B. P. A., Neves, K. do C., Fassarella, M. B., Silva, A. C. S. da, \& Farias, B. S. (2020). Protagonização do médico na segurança do paciente nas reações transfusionais: uma revisão integrativa. Research, Society and Development, 9(7), e572974597. https://doi.org/10.33448/rsd-v9i7.4597. 
Research, Society and Development, v. 10, n. 9, e5110916635, 2021

(CC BY 4.0) | ISSN 2525-3409 | DOI: http://dx.doi.org/10.33448/rsd-v10i9.16635

Rodrigues, A. D., \& Ribeiro, L. R. (2021). Sistemas sanguíneos, incompatibilidade e procedimentos alternativos à transfusão. Brazilian Journal of Development, 7(2), 13007-13027. https://doi.org/10.34117/bjdv7n2-085.

Sahu, A., \& Bajpai, M. (2020). Determining the true incidence of acute transfusion reactions: Active surveillance at a specialized liver center. Hematology, Transfusion and Cell Therapy, 42(4), 326-332. https://doi.org/10.1016/j.htct.2019.09.006.

Santos, A. A. B. da S., Teixeira, J. D. C., Pereira, L. D. C. da S., \& Silva, A. L. de A. (2020). Conhecimento de enfermeiros sobre reações transfusionais: revisão integrativa. Revista Recien - Revista Científica de Enfermagem, 10(31), 65-73. https://doi.org/10.24276/rerecien2020.10.31.65-73.

Serious Hazards of Transfusion - SHOT. (2019). New or Unclassifiable Complications of Transfusion (UCT). Retrieved from https://www.shotuk.org/wpcontent/uploads/myimages/19.-New-or-Unclassifiable-Complications-of-Transfusion-UCT.pdf.

Simons, F. E. R., Ebisawa, M., Sanchez-Borges, M., Thong, B. Y., Worm, M., Tanno, L. K., \& Sheikh, A. (2015). 2015 update of the evidence base: World Allergy Organization anaphylaxis guidelines. World Allergy Organization Journal, 8(1), 32. https://doi.org/10.1186/s40413-015-0080-1.

Soares, F. M. M., Cruz, R. C., Almeida, R. D., Da Silva Camilo, J. K., \& Scopacasa, L. F. (2020). Avaliação dos registros de enfermagem acerca da reação transfusional. Revista Enfermagem Atual In Derme, 90(28). https://doi.org/10.31011/reaid-2019-v.90-n.28-art.511

Sobral, P. A. D. S., Göttems, L. B. D., \& Santana, L. A. (2020). Hemovigilance and patient safety: analysis of immediate transfusion reactions in elderly. Revista Brasileira de Enfermagem, 73(suppl 3), e20190735. https://doi.org/10.1590/0034-7167-2019-0735.

Souza, V. R. de, Costa, A. J. da, Costa, M. A. F. da, Dantas, S. R., Vila, L. L., \& Liparizi, S. de M. (2019). Atuação do enfermeiro frente uma reação transfusional: uma revisão bibliográfica. Arquivos Brasileiros de Medicina Naval, 80(1), 30-34. https://portaldeperiodicos.marinha.mil.br/index.php/abmn/article/download/179/211.

Suddock, J. T., \& Crookston, K. P. (2019). Transfusion Reactions. In StatPearls. Cham: Springer International Publishing. https://doi.org/10.1007/978-3-31974588-6_107.

Veiga, V. C., \& Rojas, S. S. O. (2018). Como reduzir os riscos de eventos adversos em transfusão. In Associação de Medicina Intensiva Brasileira (1st ed., pp. 59-75). Artmed Panamericana.

Vlaar, A. P. J., Toy, P., Fung, M., Looney, M. R., Juffermans, N. P., Bux, J., ... Kleinman, S. (2019). A consensus redefinition of transfusion-related acute lung injury. Transfusion, 59(7), 2465-2476. https://doi.org/10.1111/trf.15311. 